\title{
Significance of HE4 estimation in comparison with CA125 in diagnosis of ovarian cancer and assessment of treatment response
}

\author{
Elham O Hamed ${ }^{1 *}$, Hydi Ahmed ${ }^{1}$, Osama B Sedeek ${ }^{4}$, Abeer M Mohammed ${ }^{1}$, Ali A Abd-Alla² \\ and Hazem M Abdel Ghaffar ${ }^{3}$
}

\begin{abstract}
Background: Human epididymis protein 4 (HE4) is a novel and specific biomarker for ovarian cancer. The aim of this study is to evaluate a new tumor marker, HE4, in comparison with CA125 in diagnosis of epithelial ovarian cancer (EOC) and benign gynecological diseases.

Methods: CA125 and HE4 serum levels were determined in 30 patients with epithelial ovarian cancer (21 serous, 6 endometrioid and 3 mucinous tumors), 20 patients with benign gynecological diseases (8 patients with ovarian cyst, 5 patients with endometriosis, 4 patients with fibroid and 3 patients with pelvic inflammatory disease) and 20 healthy women. CA125 and HE4 cut-offs were $35 \mathrm{U} / \mathrm{ml}$ and 150 pmol/l, respectively.

Results: Serum HE4 and CA125 concentrations were significantly higher in the ovarian cancer patients compared with those seen in patients with benign disease or in the healthy controls ( $p=0.001$ and $p<0.001$ respectively). In the receiver operating characteristic analysis (ROC), the area under the curve (AUC) values for HE4 was 0.96 (95\% confidence interval, 0.9-1.0) and CA125 was 0.82 (95\% confidence interval, 0.7-0.94). Compared to CA125, HE4 had higher sensitivity (90\% vs. $83.3 \%$ ), specificity (95\% vs. $85 \%$ ), PPV (93.1\% vs. $80.7 \%$ ) and NPV ( $92.7 \%$ vs. $87.2 \%)$, the combination of HE4 + CA125 the sensitivity and PPV reached $96.7 \%$ and $97 \%$ respectively.
\end{abstract}

Conclusion: Measuring serum HE4 concentrations along with CA125 concentrations may provide higher accuracy for detecting epithelial ovarian cancer.

Virtual Slides: The virtual slide(s) for this article can be found here: http://www.diagnosticpathology.diagnomx.eu/ vs/1060413168685759

Keywords: Human epididymis protein 4, HE4, Carbohydrate antigen125, CA125, Epithelial, Ovarian cancer, EOC

\section{Introduction}

The annual incidence of ovarian cancer is 204,000, with 125,000 deaths. In developed countries, ovarian cancer remains the most lethal of all gynecologic malignancies. One of the reasons for the high fatality rate is that more than $70 \%$ of women with ovarian cancer are diagnosed with advanced disease. There is a close correlation between stage at presentation and survival; therefore, early detection of ovarian cancer represents the best hope for mortality reduction and long term disease control. There

\footnotetext{
* Correspondence: elhamomar@yahoo.com

'Clinical Pathology Department, Faculty of Medicine, Sohag University, Sohag 82524, Egypt

Full list of author information is available at the end of the article
}

is preliminary evidence that screening can improve survival, but the impact of screening on mortality from ovarian cancer is still unclear [1].

Epithelial ovarian cancer set by the World Health Organization (WHO) recognizes eight histological tumor subtypes: serous, mucinous, endometrioid, clear cell, transitional cell, squamous cell, mixed epithelial and undifferentiated. Within each subtype, tumors are further described as benign, malignant, or borderline, and depending upon tumor subtype; classified as low or high-grade. Borderline tumors are considered to have low malignant potential and/or indolent behavior [2].

Serous tumors, which carry the poorest prognosis, are the most common form of ovarian carcinoma and make

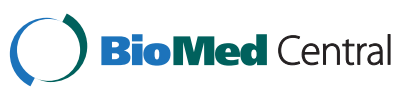


up $30-70 \%$ of all diagnoses. Serous tumors are histologically similar to cancers of the fallopian tube, and range from cystic papillary tumors to solid masses. Endometrioid tumors, accounting for $10-20 \%$ of ovarian carcinomas, are characterized by endometrial-like glandular structures. Mucinous tumors often contain cysts and glands lined by mucin-rich cells and constitute $5-20 \%$ of ovarian carcinomas. Clear cell tumors represent 3-10\% of ovarian carcinomas and are comprised of clear and hobnailed cells with an immature glomerular pattern. Undifferentiated carcinomas constitute 1\% [3].

Recent morphologic, immunohistochemical, and molecular genetic studies have led to the development of a new paradigm for the pathogenesis and origin of epithelial ovarian cancer based on a dualistic model of carcinogenesis that divides epithelial ovarian cancer into 2 broad categories designated types I and II. Type I tumors include all major histotypes (serous, endometrioid, mucinous, clear cell, and transitional) but exhibit low-grade nuclear and architectural features, slow growth, and can be linked to well-defined benign ovarian precursor lesions. They are generally indolent, present in stage I (tumor confined to the ovary), and are characterized by specific mutations, including KRAS, BRAF, ERBB2, CTNNB1, PTEN, PIK3CA, ARID1A, and PPP2R1A. Type II tumors comprise highgrade serous, high-grade endometrioid, malignant mixed mesodermal tumors (carcinosarcomas), and undifferentiated carcinomas. They are aggressive, present in advanced stage, and have a very high frequency of TP53 mutations and may also exhibit gene amplification and overexpression of HER2/neu and AKT2 oncogenes [4]. Several protooncogenes, tumor suppressor genes, and apoptosis related genes including bax, bcl-2, p53, p21, myc, c-kit, telomerase, and metallothionein have been investigated in ovarian tumors which can reliably predict the rate of progression and the response to chemotherapy and can facilitate ovarian cancer typing [5-8]. Recent Immunohistochemical staining of ovarian cancer for potassium channels (Kv1.3, K2p9.1, Eag and HERG) have been shown to be overexpressed in ovarian cancer where they appear to play a role in cell proliferation and progression [9].

Symptoms of epithelial ovarian cancer are often nonspecific, especially in early stage cancer. Ultrasound is used to assess patients for ovarian cancer; ultrasound has a low specificity for determining if a mass is benign or malignant. The specificity is improved by using Doppler ultrasound and a morphology index but performance varies amongst different operators [10].

The use of tumor markers to further characterize the mass has come into clinical use. Carbohydrate antigen 125 (CA125) is the most widely used tumor marker in ovarian cancer; however, its predictive power is far from ideal. It is elevated in about $80 \%$ of women with epithelial ovarian cancer (EOC) but only in $50 \%$ of women with early stage disease [11]. The specificity of CA125 is limited, since it can be elevated in a range of common benign gynecologic or non-gynecologic conditions [12]. Furthermore, the sensitivity and specificity of CA125 are not high enough for population screening for the detection of early stage ovarian cancer [13].

The identification of new cancer biomarkers to replace or complement CA125 is urgently needed and currently underway. Accordingly, there have been many efforts to improve the diagnostic performance of markers or marker combinations, and some markers, including mesothelin, CA72-4, inhibin, kallikreins, and osteopontin, have been investigated to complement CA125 and to improve its sensitivity for early detection [12,14]. Among these, human epididymis protein 4 (HE4), also known as WAPtype four disulphide core 2 (WFDC2), is one of the most promising markers for improving the sensitivity and specificity. HE4 is primarily expressed in the reproductive and respiratory tracts [15] and is overexpressed in ovarian cancer cells, especially in histologic subtypes of serous or endometriod carcinoma [16] and it has been suggested to be a serological marker of ovarian cancer [17]. In this study, we aimed to compare the characteristics of HE4 and CA125 in epithelial ovarian cancer and benign gynecological diseases, and to evaluate the diagnostic performance of both CA125 and HE4 in discriminating ovarian cancer from other benign gynecologic diseases.

\section{Methods}

\section{Subjects and study design}

In this study, 30 female patients were selected with recently diagnosed malignant epithelial ovarian cancers, who consecutively were admitted at Oncology department in Sohag University from March 2011 to July 2012. The inclusion criteria were: availability of complete clinical records, informed consent and agreement to have additional testing for new markers, clinical and histological diagnosis with staging and grading of ovarian cancer, according to the current classification and guidelines. The mean age was $50.7 \pm 14.6$ years (range 24-73), and 9 were in menopause (30\%). The histological diagnosis was as follows: 21 serous (70\%), 6 endometrioid (20\%) and 3 mucinous tumors (10\%). It was approved by the faculty committee for research ethics. The exclusion criteria: were pregnancy and significant concomitant diseases such as chronic heart failure, and severe chronic liver or renal disease. The second group consisted of 20 patients with benign gynecological diseases, with a mean age of $45.1 \pm$ 14.7 years (range 40-60) and 5 in menopause (25\%). The group with benign diseases included 8 patients with ovarian cyst (40\%), 5 patients with endometriosis (25\%), 4 patients with fibroid (20\%) and 3 patients with pelvic inflammatory disease (15\%). The third group included 
20 apparently healthy females matched for the same age group as a control group.

\section{Methods}

Venous blood samples were collected following an overnight fasting (serum and EDTA samples) before chemotherapy treatment and at 6 month intervals thereafter. Serum glucose, liver function tests and renal function testes were analyzed on autoanalyzer Cobas c 311 (Roche/ Hitachi cobas C systems). Complete blood picture on cell dyne-2700 fully automated cell counter. CA125 analysis was done on Axsym system (Abbott Diagnostics Division, Chicago) based on Microparticle Enzyme Immunoassay (MEIA) technology. HE4 assay was performed on the fully automated ARCHITECT instrument (Abbott Diagnostics Division, Chicago) based on Chemiluminescentmicroparticle immunoassay (CMIA). The two monoclonal antibodies (2 $\mathrm{H} 5$ and 3D8) were used for capture and detection of HE4. In the first step, sample and $2 \mathrm{H} 5$ anti-HE4 coated paramagnetic microparticles are combined. HE4 antigen present in the sample binds to the anti-HE4 coated microparticles. After washing, 3D8 anti-HE4 acridinium labeled conjugate is added to create a reaction mixture in the second step. Following another wash cycle, pre-trigger and trigger solutions are added to the reaction mixture. The resulting chemiluminescent reaction is measured as relative light units. A direct relationship exists between the amount of HE4 antigen in the sample and the relative light units.

\section{Statistical analysis}

The data are presented as mean \pm standard deviation (SD) or median (range) and number (n). Linear relationships between variables were determined using Spearman's rank correlation test. One way analysis of variance (ANOVA) test was used followed by post hoc test to determine the significance of variables when comparing more than 2 groups. Non-parametric receiver operating characteristic (ROC) analyses were performed to evaluate diagnostic values of individual parameters generated by graphically plotting sensitivity versus specificity with using 95\% confidence intervals (CI). The diagnostic accuracy of the test is measured by the area under the curve (AUC). Statistical significance is considered a value of $P<0.05$. All statistical analyses were performed using SPSS software, version 10.0.

\section{Results}

Clinical characteristics and laboratory variables of the studied groups were demonstrated in Table 1 . The median CA125 and HE4 levels in the healthy control were $12.5 \mathrm{U} / \mathrm{ml}$ and $56.9 \mathrm{pmol} / \mathrm{l}$ respectively. There were significant difference in HE4 \& CA125 values between the ovarian cancer group (median $295.5 \mathrm{U} / \mathrm{ml}$, for CA125, and median $237.2 \mathrm{pmol} / \mathrm{l}$, for HE4) than the benign gynecological disease (median $26.9 \mathrm{U} / \mathrm{ml}, \mathrm{p}=0.001$ for CA125, and median $66.1 \mathrm{pmol} / \mathrm{l}, \mathrm{p}=0.001$ for HE4) and control group $(\mathrm{p}<0.001)$. CA125 was also higher in

Table 1 Clinical and laboratory variables of studied groups

\begin{tabular}{|c|c|c|c|c|}
\hline & Ovarian cancer $(n=30)$ & Benign gynecological $(n=20)$ & Controls $(n=20)$ & $p$ (ANOVA) \\
\hline Age (range) & $24-73$ & $40-60$ & $30-66$ & - \\
\hline Menopause\% & 30 & 25 & 20 & - \\
\hline RBCs count $\times 10^{12} / /$ & $4.5 \pm 0.5$ & $4.5 \pm 0.49$ & $4.8 \pm 0.5$ & NS \\
\hline $\mathrm{Hb} \mathrm{g} / \mathrm{dl}$ & $12.1 \pm 1.5$ & $12.5 \pm 1.4$ & $12 \pm 1.3$ & NS \\
\hline WBCs $\times 10^{9} / 1$ & $8.1 \pm 2.9$ & $6.9 \pm 2.3$ & $7.6 \pm 2$ & NS \\
\hline Platelets $\times 10^{9} / 1$ & $270 \pm 82$ & $294.7 \pm 72.1$ & $264.7 \pm 59.4$ & NS \\
\hline S.glucose (mg/dl) & $95 \pm 15.4$ & $91 \pm 7.4$ & $85 \pm 9.4$ & NS \\
\hline S.urea (mg/dl) & $31.4 \pm 8.9$ & $29.1 \pm 6.7$ & $27.9 \pm 7.9$ & NS \\
\hline S.creatinine (mg/dl) & $0.7 \pm 0.15$ & $0.7 \pm 0.13$ & $0.73 \pm 0.12$ & NS \\
\hline $\mathrm{ALT}(\mathrm{U} / \mathrm{I})$ & $17.4 \pm 12.6$ & $15.4 \pm 11.5$ & $13.9 \pm 7.2$ & NS \\
\hline AST (U/I) & $24.7 \pm 14.5$ & $21.1 \pm 10.4$ & $21.1 \pm 6.1$ & NS \\
\hline ALP (U/I) & $76.4 \pm 24.2$ & $68.5 \pm 10.9$ & $70.5 \pm 11.3$ & NS \\
\hline Total protein $\mathrm{g} / \mathrm{dl}$ & $7.6 \pm 0.6$ & $7.4 \pm 0.5$ & $7.7 \pm 0.53$ & NS \\
\hline Albumin $\mathrm{g} / \mathrm{dl}$ & $3.8 \pm 0.33$ & $3.9 \pm 0.36$ & $3.9 \pm 0.29$ & NS \\
\hline Total bilirubin mg/dl & $0.46 \pm 0.3$ & $0.6 \pm 0.26$ & $0.5 \pm 0.24$ & NS \\
\hline D. bilirubin mg/dl & $0.12 \pm 0.07$ & $0.11 \pm 0.06$ & $0.1 \pm 0.06$ & NS \\
\hline CA125 U/ml & $295.5(4.2-1781)^{\mathrm{b}, \mathrm{c}}$ & $26.9(9.4-553)^{a}$ & $12.5(4.1-34.3)$ & $<0.001$ \\
\hline HE4 pmol// & $237.2(34.3-4090)^{b, c}$ & $66.1(24.8-179.2)$ & $56.9(20.8-111.6)$ & $<0.001$ \\
\hline
\end{tabular}

Values are expressed as the mean \pm standard deviation or median (range). ${ }^{a} \mathrm{P}<0.05,{ }^{b} \mathrm{P}<0.001$, with respect to the control group; ${ }^{\mathrm{c}} \mathrm{P}=0.001$, with respect to benign gynecological dis. 
patients with benign gynecologic diseases $(\mathrm{p}=0.04)$ than in the healthy control, but HE4 was not ( $\mathrm{p}>0.05)$. Table 2 showed FIGO stage of the studied woman, $40 \%$ of the patients had stage 3 and the serum levels of HE4 and CA125 in relation to histological types with higher significant level in serous ovarian cancer $(\mathrm{p}<0.01$ and $\mathrm{p}<0.05$ respectively). In 30 women with EOC, HE4 were significantly higher than CA125 in sensitivity and specificity ( $90 \%$ vs $83.3 \%$ and $95 \%$ vs. $85 \%$, respectively). Also, the, positive predictive values (PPV) and negative predictive values (NPV) for HE4 were significantly higher than CA125 (93.1\% vs. $80.7 \%$ and $92.7 \%$ vs. $87.2 \%$, respectively). Sensitivity and PPV were increased reached $96.7 \%$, 97\% respectively when the two markers combined with each other (Table 3). Figure 1 shows the receiver operating characteristic analysis (ROC) plot for all women with epithelial ovarian cancer and benign diseases, the area under the curve (AUC) for CA125 was 0.82 (95\% CI 0.7-0.94) and for HE4 was 0.96 (95\% CI 0.9-1.0) ( $<<0.01)$ for distinguishing between EOC and benign disease. A positive correlation between serum levels of HE4 and CA125 was observed in women with epithelial ovarian cancer, benign gynecological disease group and control group $(\mathrm{r}=0.5, \mathrm{p}<0.01)$ (Figure 2). Of the 30 patients in the study, three were resistant to treatment. The remaining 27 patients achieved remission determined by normalization of CA125 and HE4 levels, physical examination and imaging by computerized tomography (CT) magnetic resonance imaging (MRI) or ultrasound. Table 4 (Figure 3): show significant difference $(\mathrm{P}<0.001)$ for CA125 and HE4 levels before and after chemotherapy. In the three patients with persistent disease following chemotherapy and the presence of tumor confirmed by imaging results (one patient with omental deposit and two patients with residual tissues), CA125 and HE4 dropped but remained above their respective thresholds thereafter.

\section{Discussion}

Despite the development of new treatments and therapies designed to improve the five year survival rate, ovarian cancer still remains the deadliest cancer of the female reproductive tract. Five-year survival rate is $90 \%$

Table 2 HE4 \& CA125 serum levels in patients with EOC according to tumor stage and histological type

\begin{tabular}{llll}
\hline & $\mathbf{n}$ & HE4 median (range) & CA125 median (range) \\
\hline Stage I-II & 8 & $210(55-1060)$ & $36(4.2-410)$ \\
Stage III & 12 & $315(34.3-2817)$ & $120(26-1300)$ \\
Stage IV & 10 & $491(45-4090)$ & $320(150-1781)$ \\
Serous & 21 & $305(57-4090)$ & $285(47-1781)$ \\
Endometrioid & 6 & $170(34.3-1650)$ & $137(35-1011)$ \\
Mucinous & 3 & $159(142-410)$ & $66(4.2-405)$ \\
\hline
\end{tabular}

Table 3 Sensitivity, specificity, positive and negative predictive values (PPV and NPV) for CA125, HE4, and in combination with each other

\begin{tabular}{lllll}
\hline & Sensitivity & Specificity & PPV & NPV \\
\hline HE4 & $90 \%$ & $95 \%$ & $93.1 \%$ & $92.7 \%$ \\
CA125 & $83.3 \%$ & $85 \%$ & $80.7 \%$ & $87.2 \%$ \\
HE4 + CA125 & $96.7 \%$ & $80 \%$ & $97 \%$ & $80 \%$ \\
\hline
\end{tabular}

when disease is confined to the ovaries but overall survival is poor because only $25 \%$ of cases are found in this early stage. Unfortunately, most cases are diagnosed in the late stages of the disease, when the five-year survival rates fall below $20 \%$, with most patients having metastatic disease at presentation. This further contributes to worsening the prognosis. The lack of precise early warning signs is one of the factors that further contribute to the fact that only $25 \%$ of ovarian tumors are identified at stage I [18].

CA125 is still the only tumor marker recommended as a diagnostic or prognostic indicator and for the monitoring of disease recurrence after surgery and adjuvant chemotherapy [19-21]. The major drawback of CA125 is the documented lack of specificity, as this marker may show levels exceeding the 95th percentile of normal values in a significant proportion of women with benign or malignant diseases [22].

Accordingly, there have been many efforts to improve the diagnostic performance of CA125. Among those, a prominent relevance has been recently attributed to the HE4 which is one of the most promising marker for improving the sensitivity and specificity [23].

In this study, we investigated the role of HE4 alone and in combination with CA125 in assessing patients with epithelial ovarian cancer, regardless of the menopausal status. Initial results on HE4 testing of this study confirm the high sensitivity and specificity of this molecule over CA125 for EOC (90\% vs. $83.3 \%$ and $95 \%$ vs. $85 \%$, respectively). In our experience, no false positive results for HE4 or CA125 were recorded on healthy women, the specificity was more evident among patients with benign gynecological lesions, since only 2 were positive for HE4, while 6 were positive for CA125 (10\% vs $30 \%$ ). we found a significant difference in CA125 values between the benign gynecological disease group and control group $(\mathrm{p}<0.05)$. Unlike CA125, HE4 was shown not to be elevated in endometriosis $[24,25]$ and this may contribute to the improved performance with HE4.

The diagnostic performance of CA125 and HE4 in discriminating ovarian cancer from healthy and benign gynecologic conditions was verified using ROC analysis. The resultant AUC values were 0.96 for HE4 (95\% CI $0.90-1.0)$ and 0.82 for CA125 (95\% CI 0.70-0.94) ( $<<0.01)$, which would make them feasible for use as tumor markers, 


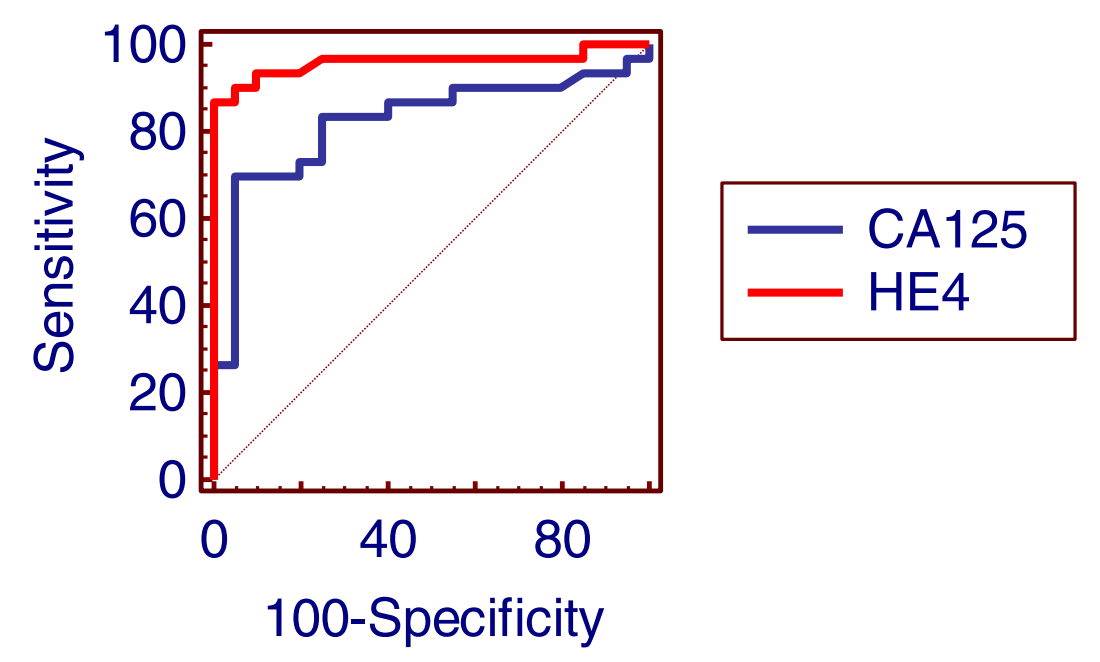

\begin{tabular}{|l|l|}
\hline Marker & AUC (95\% CI) \\
\hline HE4 & $0.96(0.90-1)$ \\
\hline CA125 & $0.82(0.70-0.94)$ \\
\hline p & $<0.01$ \\
\hline
\end{tabular}

Figure 1 ROC plot and AUC for CA125 and HE4 for patients with epithelial ovarian cancer and benign diseases.

have also investigated the usefulness of HE4 in to differentiate ovarian cancers from healthy and benign gynecologic conditions. This finding is in agreement with several researchers who reported that HE4 has a clear specificity edge over CA125 and also a better sensitivity for EOC, in general and in the early stages patients [26-30].

HE4 as well as CA125 are not only found in ovarian carcinoma; abnormal levels may be also found in some benign conditions or other gynecologic and non-gynecologic malignancies; for example, breast, pancreatic, and endometrial cancers. These results imply that HE4 and CA125 are not especially specific to ovarian cancer [31].

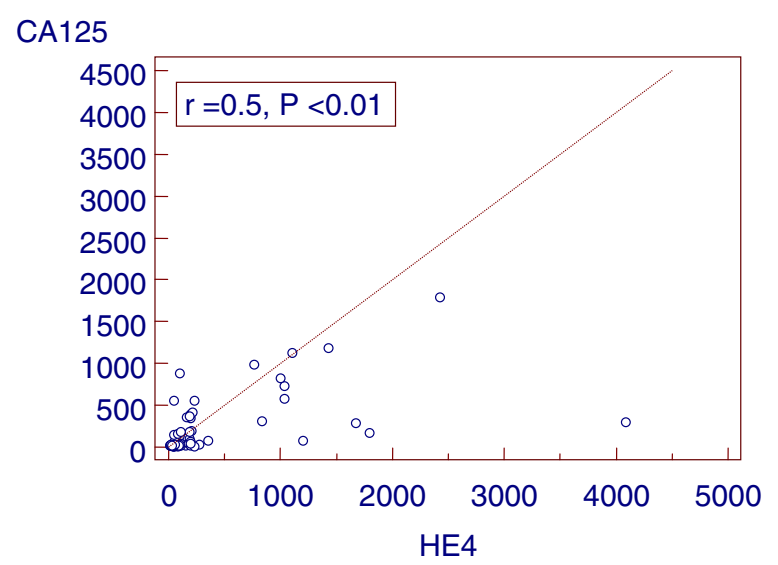

Figure 2 Correlation (Spearman) between HE4 and CA125 on all groups.
Another study demonstrated that HE4 might be associated with the innate immune defenses of the lung, nasal and oral cavities [32]. Several researchers have also investigated the usefulness of HE4 in other malignancies, including transitional cell carcinoma of the urinary tract and endometrial cancer [33].

The limits of any single tumor marker for EOC have been addressed in several recent experiences, in which a multimarker approach has been pursued in order to achieve a better diagnostic accuracy [34-38]. Combined the two markers showed improved sensitivity to $96.7 \%$, and increased PPV. There was a $12.4 \%$ increase in PPV for CA125 compared to HE4 (16.3\% vs. 3.9\%). Improving the PPV not only means less inappropriate referrals and its associated costs, but also a reduction in the number of midline laparotomies, which is still the standard for cares for women with suspected EOC.

The correlation between HE4 and CA125 levels was estimated using Spearman's rank correlation test, most of the measured values tended to be increased for both markers. However, the degree of correlation was not so strong $(r=0.5)$, and there were some discordant results. These mean that each marker was elevated concurrently

Table 4 Levels of HE4 and CA125 in the ovarian cancer group before and after treatment

\begin{tabular}{llll}
\hline & Before treatment & After treatment & P value \\
\hline HE4 & $237.2(34.3-4090)$ & $91.9(45.4-180)$ & $<0.001$ \\
CA125 & $295.5(4.2-1781)$ & $11.6(4-71.5)$ & $<0.001$ \\
\hline
\end{tabular}




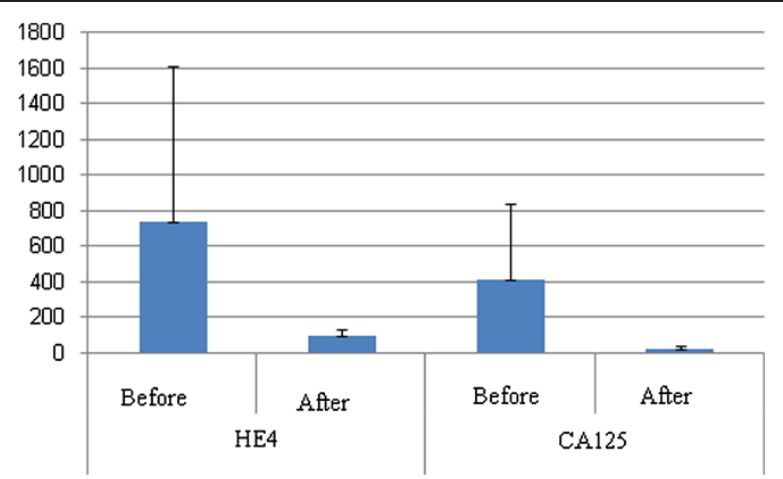

Figure 3 HE4 and CA125 in the ovarian cancer group before and after treatment.

or under some different conditions, and these also support the necessity of combining the two markers.

The increases in both markers were more evident in certain histologies of ovarian cancer than in others. Galgano et al. reported that HE4 proteins or genes were expressed strongly in serous papillary, clear cell, and endometrioid carcinoma of the ovaries. However, other histologies of ovary cancer or non-gynecologic malignancies including invasive ductal carcinoma of breast, endometrial, pancreaticobiliary, and renal cell carcinoma also exhibited strong or weak expressions of HE4 proteins [15]. In our result, the predominant histological type observed is serous followed by endometriod and mucinous, we found both tumor markers HE4 and CA125 were related to tumor stage and histological types with the lowest concentration in mucinous subtype (median $66 \mathrm{U} / \mathrm{ml}$, for CA125, and median 159 $\mathrm{pmol} / \mathrm{l}$, for HE4) and elevation of HE4 or CA125 was obvious in serous subtypes (median $285 \mathrm{U} / \mathrm{ml}$, for CA125, and median $305 \mathrm{pmol} / \mathrm{l}$, for HE4) but was not evident in other histological type. Kobel et al. investigated the variation in expression of 21 different markers in accordance with ovarian cancer subtypes, and concluded that most markers differ significantly between histological types [39].

In assessment of treatment response both CA125 and HE4 levels show significant difference before and after chemotherapy $(\mathrm{P}<0.001)$ for both, in which normalization of CA125 and HE4 levels occurred. Relevant thresholds for using these markers for remission monitoring have not been established. There is an established threshold range for various commercial CA125 assays [40]. Thresholds for HE4 have been reported only recently but remain uncertain [41-43]. In three patients CA125 and HE4 dropped but remained above their respective thresholds thereafter (one patient had high CA125 and others had high HE4). HE4 serum levels are related to progression of disease stage [44] and hence to tumor burden. A failure of HE4 levels to normalize at the completion of primary therapy could be related to persistent disease not detected by
CA125 nor by physical exam or CT imaging. These patients may represent a high risk group who could potentially benefit from additional treatment or more intensive monitoring. Confirmation of this HE4 behavior in a larger number of patients is therefore required.

In addition to the search of specific markers for the determination of the early stages of any cancer form, it is just as important to find markers capable of following the remission from disease as response to therapy. The suggestion that HE4 is a good indicator for the remission from the disease was reported by a follow up study by Allard et al. in which it was shown that the values of HE4 correlated with the clinical response to treatment or remission from the disease, as documented by CT imaging [45].

\section{Conclusion}

HE4 demonstrated comparable diagnostic performances to CA125 as a tumor marker for detecting ovarian cancer. HE4 was more sensitive in detecting early stages of ovarian cancer and more specific. HE4 improves the utility of CA125 as a tumor marker in ovarian cancer, and using both markers simultaneously increases the tumor marker sensitivity. The use of this combination might enable to improve detection of ovarian cancer as compared with use of either marker alone for the discrimination of benign from malignant ovarian lesions.

\section{Abbreviations}

HE4: Human epididymis protein 4; CA125: Carbohydrate antigen125; EOC: Epithelial ovarian cancer; PPV: Positive predictive value; NPV: Negative predictive value; ROC: Receiver operating characteristic analysis; AUC: Area under the curve; RBCs: Red blood cells; WBCs: White blood cells; ALT: Alanine transaminase; AST: Aspartate transaminase; ALP: Alkaline phosphatase.

\section{Competing interest}

The authors declare that they have no conflict of interest and did not receive any financial support from any source outside the university.

\section{Authors' contributions}

I and my Clinical Pathology colleagues have tested the serum markers either CA125 or HE4 besides routine investigation and prepared the introduction, results and discussion of this article. My colleague from the Obstetrics \& Gynecology department has provided us with the specimens from patients and shared in the discussion of this article. My colleague from the clinical oncology department has made and commented on the clinical, histological diagnosis with staging and grading of ovarian cancer, CT examination of the patient and chemotherapy treatment. All authors read and approved the final manuscript.

\section{Acknowledgements}

To my Husband Tarek Abulezz MD who helped me in submitting this paper and also to all members of Clinical Pathology Dept., Sohag University.

\section{Author details}

'Clinical Pathology Department, Faculty of Medicine, Sohag University, Sohag 82524, Egypt. ${ }^{2}$ Clinical Oncology Department, Faculty of Medicine, Sohag University, Sohag 82524, Egypt. ${ }^{3}$ Obstetrics \& Gynecology Department, Faculty of Medicine, Sohag University, Sohag, Egypt. ${ }^{4}$ Clinical Pathology Department, Faculty of Medicine, Assiut University, Assiut, Egypt.

Received: 14 December 2012 Accepted: 8 January 2013

Published: 23 January 2013 


\section{References}

1. Rauh-Hain JA, Krivak TC, del Carmen MG, Olawaiye AB: Ovarian cancer screening and early detection in the general population. Rev Obstet Gynecol 2011, 4(1):15-21.

2. Lee KR, Tavassoli FA, Prat J, Dietel M, Gersell DJ, Karseladze Al, Hauptmann S, Rutgers J: WHO histological classification of tumours of the ovary (chapter 2). In pathology and genetics of tumours of the breast and female genital organs. Edited by Tavassoli FA, Devilee P. Lyon: IARC Pres; 2003:113-161.

3. Nolen B, Marrangoni A, Velikokhatnaya L, Prosser D, Winans M, Gorelik E, Anna LA: A serum based analysis of ovarian epithelial tumorigenesis. Gynecol Oncol 2009, 112(1):47-54

4. Kurman RJ, Shih I-M: Molecular pathogenesis and extraovarian origin of epithelial ovarian cancer-shifting the paradigm. Hum Pathol 2011, 42(7):918-931.

5. Hatice O, Goncalmir Y, Sema A, Meral C, Ersin T, Ali C: Immunohistochemistry with apoptotic-antiapoptotic proteins (p53, p21, bax, bcl-2), c-kit, telomerase, and metallothionein as a diagnostic aid in benign, borderline, and malignant serous and mucinous ovarian tumors. Diagn Pathol 2012, 7:124.

6. Fauvet R, Dufornet C, Poncelet C, Uzan C, Hugol D, Darai E: Expression of Pro apoptotic (p53, p21, bax, bak and fas) and anti-apoptotic (bcl-2 and bcl-x) proteins in serous versus mucinous borderline ovarian tumours. J SurgOncol 2005, 92:337-343.

7. Nielsen JS, Jakobsen E, Holund B, Bertelsen K, Jakobsen A: Prognostic significance of p53, Her-2, and EGFR overexpression in borderline and epithelial ovarian cancer. Int J Gynecol Cancer 2004, 14:1086-1096.

8. Havrilevsky L, Darcy K, Hamdan R, Priore RL, Leon J, Bell J, Berchuck A: Prognostic significance of p53 mutation and p53 overerpression in advanced epithelial ovarian cancer: a gynecologic oncology group study. J Clin Oncol 2003, 21:3814-3825.

9. Viren A, Raheela K, Averil W, Robert S, Schalkwyk GV, Anish B, Sowter HM: The Eag potassium channel as a new prognostic marker in ovarian cancer. Diagn Pathol 2010, 5:78.

10. Valentin L: Use of morphology to characterize and manage common adnexal masses. Best Pract Res Clin Obstet Gynaecol 2004, 18:71-89.

11. Rosen DG, Wang L, Atkinson JN, et al: Potential markers that complement expression of CA125 in epithelial ovarian cancer. Gynecol Oncol 2005, 99:267-277.

12. Maggino T, Gadducci A, D'Addario V, et al: Prospective multicenter study on CA125 in postmenopausal pelvic masses. Gynecol Oncol 1994, 54:117-123.

13. Kobayashi H, Yamada Y, Sado T, et al: A randomized study of screening for ovarian cancer: a multicenter study in Japan. Int I Gynecol Cancer 2008, 18:414-420.

14. Yousef GM, Polymeris ME, Yacoub GM, et al: Parallel overexpression of seven kallikrein genes in ovarian cancer. Cancer Res 2003, 63:2223-2227.

15. Galgano MT, Hampton GM, Frierson HF Jr: Comprehensive analysis of HE4 expression in normal and malignant human tissues. Mod Pathol 2006, 19:847-853.

16. Drapkin $R$, von Horsten $H H$, Lin $Y$, et al: Human epididymis protein 4 (HE4) is a secreted glycoprotein that is overexpressed by serous and endometrioid ovarian carcinomas. Cancer Res 2005, 65:2162-2169.

17. Ruggeri G, Bandiera E, Zanotti L, Belloli S, Ravaggi A, Romani C, et al: HE4 and epithelial ovarian cancer: comparison and clinical evaluation of two immunoassays and a combination algorithm. Clin Chim Acta 2011, 412:1447-1453.

18. Rien BJD, Gupta S, Dada R, Safi J, Michener C, Agarwal A: Potential markers for detection and monitoring of ovarian cancer. J Oncology 2011, 2011:475983.

19. Aebi S, Castiglione M: Newly and relapsed epithelial ovarian carcinoma: ESMO Clinical Recommendations for diagnosis, treatment and follow-up. Ann Oncol 2009, 20(Suppl 4):21-23.

20. Sturgeon CM, Duffy MJ, Stenman U-H, et al: National academy of clinical biochemistry laboratory medicine practice guidelines for use of tumor markers in testicular, prostate, colorectal, breast, and ovarian cancers. Clin Chem 2008, 54:e11-e79.

21. Bast RC Jr: Status of tumor markers in ovarian cancer screening. J Clin Oncol 2003, 21:200-205.

22. Moore RG, Jaube-Raughley M, Brown AK, et al: Comparison of a novel multiple marker assay vs. The risk of malignancy index for the prediction of epithelial ovarian cancer in patients with a pelvic mass. Am J Obstet Gynecol 2010, 203:e1-e6.

23. Kim YM, Whang DH, Park J, Kim SH, Lee SW, Park HA, Ha M, Choi KH: Evaluation of the accuracy of serum human epididymis protein 4 in combination with CA125 for detecting ovarian cancer: a prospective case-control study in a Korean population. Clin Chem Lab Med 2011, 49(3):527-534

24. Huhtinen K, Suvitie P, Hiissa J, Junnila J, Huvila J, Kujari H, et al: Serum HE4 concentration differentiates malignant ovarian tumours from ovarian endometriotic cysts. Br J Cancer 2009, 100:1315-1319.

25. Bordin L, Fiore C, Donà G, Andrisani A, Ambrosini G, Faggian D, et al: Evaluation of erythrocyte band 3 phosphotyrosine level, glutathione content, CA-125, and human epididymal secretory protein E4 as combined parameters in endometriosis. Fertil Steril 2010, 94:1616-1621.

26. Li J, Dowdy S, Tipton T, et al: HE4 as a biomarker for ovarian and endometrial cancer management. Expert Rev Mol Diagn 2009, 9:555-566.

27. Montagnana M, Lippi G, Ruzzenente $\mathrm{O}$, et al: The utility of serum human epididymis protein 4 (HE4) in patients with a pelvic mass. Clin Lab Anal 2009, 23:331-335.

28. Gorp T, Cadron I, Despierre E, Daemen A, Leunen K, Amant F, et al: HE4 and CA125 as a diagnostic test in ovarian cancer: prospective validation of the risk of ovarian malignancy algorithm. Br J Cancer 2011, 104(5):863-870.

29. Montagnana M, Danese E, Ruzzenente O, Bresciani V, Nuzzo T, Gelati M, et al: The ROMA (Risk of Ovarian Malignancy Algorithm) for estimating the risk of epithelial ovarian cancer in women presenting with pelvic mass: is it really useful? Clin Chem Lab Med 2011, 49(3):521-525.

30. Rafael M, Escudero JM, Augé JM, Xavier F, Laura F, Aureli T, Jose L, Jaume P: HE4 a novel tumour marker for ovarian cancer: comparison with CA125 and ROMA algorithm in patients with gynecological diseases. Tumour Biol 2011, 32(6):1087-1095.

31. Park Y, Lee JH, Hong DJ, Lee EY, Kim HS: Diagnostic performances of HE4 and CA125 for the detection of ovarian cancer from patients with various gynecologic and non-gynecologic diseases. Clin Biochem 2011 44:884-888

32. Bingle L, Cross SS, High AS, et al: WFDC2 (HE4): a potential role in the innate immunity of the oral cavity and respiratory tract and the development of adenocarcinomas of the lung. Respir Res 2006, 7:61.

33. $\mathrm{Xi}$ Z, LinLin M, Ye T: Human epididymis protein 4 is a biomarker for transitional cell carcinoma in the urinary system. J Clin Lab Anal 2009, 23:357-361.

34. Moore RG, Brown AK, Miller MC, et al: The use of multiple novel tumor biomarkers for the detection of ovarian carcinoma in patients with a pelvic mass. Gynecol Oncol 2008, 108:402-408.

35. Moore RG, McMeekin DS, Brown AK, et al: A novel multiple marker bioassay utilizing HE4 and CA125 for the prediction of ovarian cancer in patients with a pelvic mass. Gynecol Oncol 2009, 112:40-46.

36. Havrilesky $L$, Whitehead CM, Rubatt JM, et al: Evaluation of biomarker panels for early stage ovarian cancer detection and monitoring for disease recurrence. Gynecol Oncol 2008, 110:374-382.

37. Nolen B, Velikokhkatnaya L, Marrangoni A, et al: Serum biomarker panels for the discrimination of benign from malignant cases in patients with an adnexal mass. Gynecol Oncol 2010, 117:440-445.

38. Yurkovetsky Z, Skates S, Lomakin A, et al: Development of a multimarker assay for early detection of ovarian cancer. J Clin Oncol 2010, 28:2159-2166

39. Kobel M, Kalloger SE, Boyd N, et al: Ovarian carcinoma subtypes are different diseases: implications for biomarker studies. PLoS Med 2008, 5:e232.

40. Rustin GJ, Vergote I, Eisenhauer E, Pujade-Lauraine E, Quinn M, et al: Definitions for response and progression in ovarian cancer clinical trials incorporating RECIST 1.1 and CA125 agreed by the Gynecological Cancer Intergroup (GCIG). Int J Gynecol Cancer 2011, 21:419-423.

41. Park Y, Kim Y, Lee EY, Lee JH, Kim HS: Reference ranges for HE4 and CA125 in a large Asian population by automated assays and diagnostic performances for ovarian cancer. Int J Cancer 2012, 130(5):1136-1144.

42. Jacob F, Meier M, Caduff R, Goldstein D, Pochechueva T, et al: No benefit from combining HE4 and CA125 as ovarian tumor markers in a clinical setting. Gynecol Oncol 2011, 121:487-491.

43. Chang $X$, Ye $X$, Dong $L$, Cheng $H$, Cheng $Y$, et al: Human epididymis protein 4 (HE4) as a serum tumor biomarker in patients with ovarian carcinoma. Int J Gynecol Cancer 2011, 21(5):852-858. 
44. Paek J, Lee SH, Yim GW, Lee M, Kim YJ, et al: Prognostic significance of human epididymis protein 4 in epithelial ovarian cancer. Eur $J$ Obstet Gynecol Reprod Biol 2011, 158(2):338-342.

45. Allard J, Somers E, Theil R, Moore RG: Use of a novel biomarker HE4 for monitoring patients with epithelial ovarian cancer. J Clin Oncol 2008, 26:5535.

doi:10.1186/1746-1596-8-11

Cite this article as: Hamed et al:: Significance of HE4 estimation in

comparison with CA125 in diagnosis of ovarian cancer and assessment

of treatment response. Diagnostic Pathology 2013 8:11.

\section{Submit your next manuscript to BioMed Central} and take full advantage of:

- Convenient online submission

- Thorough peer review

- No space constraints or color figure charges

- Immediate publication on acceptance

- Inclusion in PubMed, CAS, Scopus and Google Scholar

- Research which is freely available for redistribution 